\title{
sciendo
}

CIVIL AND ENVIRONMENTAL ENGINEERING REPORTS

E-ISSN 2450-8594

CEER 2021; 31 (1): 0079-0092

DOI: 10.2478/ceer-2021-0006

Original Research Article

\section{DESIGNING OF STEEL CHS COLUMNS SHOWING MAXIMUM COMPRESSION RESISTANCE}

\author{
Jakub MARCINOWSKI ${ }^{1}$, Mirosław SADOWSKI ${ }^{2}$ \\ Institute of Civil Engineering, University of Zielona Góra
}

\begin{abstract}
The paper deals with a shape optimisation procedure of steel, compressed bars. Circular hollow sections (CHS) of variable cross sections and variable wall thickness are taken into account. The proposed procedure for designing of steel rods exhibiting maximum compression resistance is effective and possible to use in engineering practice. The advantage of the proposed shape of the bar is that it allows to increase the value of its load carrying capacity, i.e. it ensures the transfer of a higher value of compressive force than similar, solid struts of the same mass and length. The extent of the increase in the load capacity relative to the load capacity of the reference solid, cylindrical bar depends on the slenderness of the reference bar and ranges from $60 \%$ to $170 \%$. Due to this very beneficial fact, it can be used wherever it is required to maintain a certain stiffness and an increased value of compressive force is desired, as well as in constructions where it is necessary to reduce weight while maintaining the adopted mechanical parameters, e.g. values of load bearing capacity. Final results achieved in the research were presented in the form of the flow chart allowing to design the compressed columns of optimum shape.
\end{abstract}

Keywords: compressed bar, CHS section, shape optimization, buckling criterion, analytical approach

\footnotetext{
${ }^{1}$ ) Corresponding author: j.marcinowski@ib.uz.zgora.pl.
}

${ }^{2}$ ) Corresponding author: miroslaw.sadowski@gmail.com 


\section{INTRODUCTION}

Designing of structural systems is based primarily on meeting specific strength conditions. To this end, calculations are performed to meet load and serviceability requirements. Thanks to this, the constructor receives parameter values that allow creating a better design than others (in the indicated respects). Structural shaping, which is the result of using strictly strength conditions, may not always be satisfactory. Therefore, engineers' special attention is attracted by design optimization that goes far beyond the strength design framework (cf. [2, 15, 21]). It enables the placement of material in a given design area in such a way that the resulting load-bearing system carries the given load, and at the same time is the best possible system due to previously set criteria.

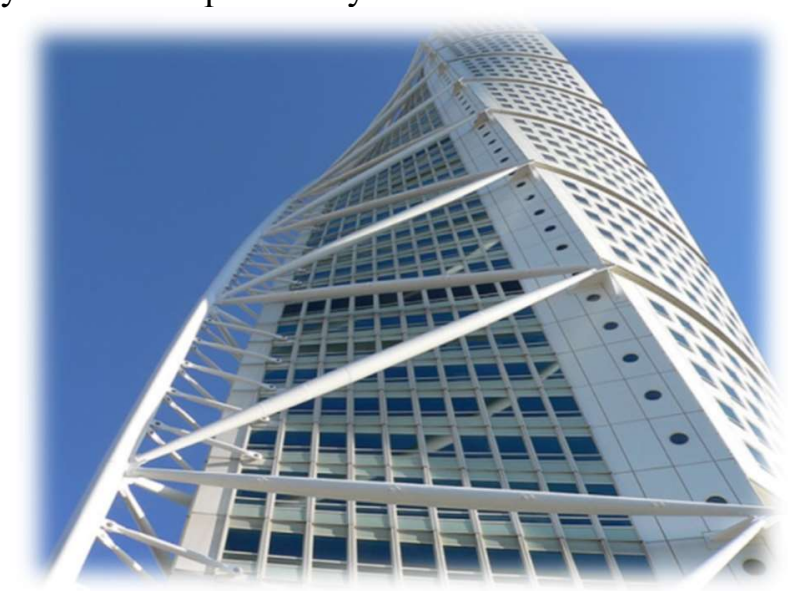

Fig. 1. Turning Torso (source: [10])

The idea described above, which has been developed almost since antiquity (cf. $[1,4,8-10,20]$ ) can be found almost everywhere - new areas of light materials applications are being discovered, e.g. in household appliances, in the construction of machinery and equipment for virtually all branches of the economy, or in construction (Fig. 1) and in vehicle construction in the broad sense: in the automotive industry, weight minimization is of great importance (Fig. 2), because the total weight of the vehicle has a significant impact on fuel consumption, which translates into operating costs and indirectly affects the emission of harmful substances into the environment.

Increasing requirements in this area determine the designers to constantly search for new, lighter materials and technical ways of joining them, as well as to shape the elements of the structure that would meet the above objectives. 


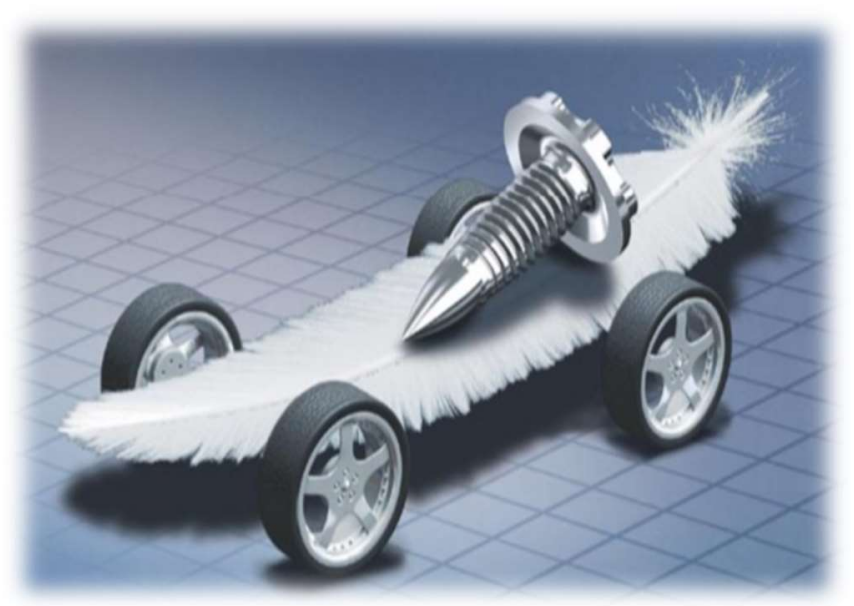

Fig. 2. The ideal image of a vehicle with minimum weight (source: [14])

The presented paper concerns the procedure of designing of steel bars with variable CHS cross-section, showing the maximum load carrying capacity. Geometrical parameters derived in the paper as a result of the optimisation procedure were expressed by closed analytical formulae and the flow chart defining consecutive steps of the designing procedure was presented as well. Compressed bars of the proposed shape can transfer forces up to $170 \%$ higher than the forces carried by solid cylindrical bars of the same mass treated in the procedure as reference bars ripening.

\section{SUBJECT OF PAPER AND MAIN ASSUMPTIONS}

The subject of detailed considerations presented in the paper is the compressed (cf. Fig. 3), non-prismatic hollow rod with an annular cross-section, the volume of which is formed in the following way: generator - external and internal - are arches of certain given continuous flat curves (the assumed curves are hyperbolic cosine - cf. description relations (2.1)), while the solid itself is the result of the rotation of these curves around the geometrical axis of the bar (see Fig. 4). Hyperbolic cosine shapes were selected between other smooth functions considered earlier (cf. [9-11]).

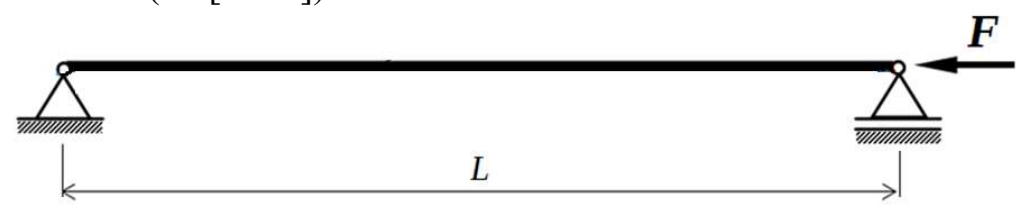

Fig. 3. Static scheme of the strut 
The bar geometry is as follows: $r_{p}$ is the length of the outer radius forming the cross-section at the rod ends, $r_{m}$ - the length of the outer radius forming the crosssection in the middle of the rod length, $\mathrm{L}$ - rod length, while $\mathrm{t}$ - wall thickness in the half rod length. The wall thickness of the bar is variable along its axis, with its ends being expressed by a compound $\alpha$ t where $\alpha$ is the ratio of the wall thickness of the bar at its ends to the wall thickness of the bar in half its length (Fig. 4).

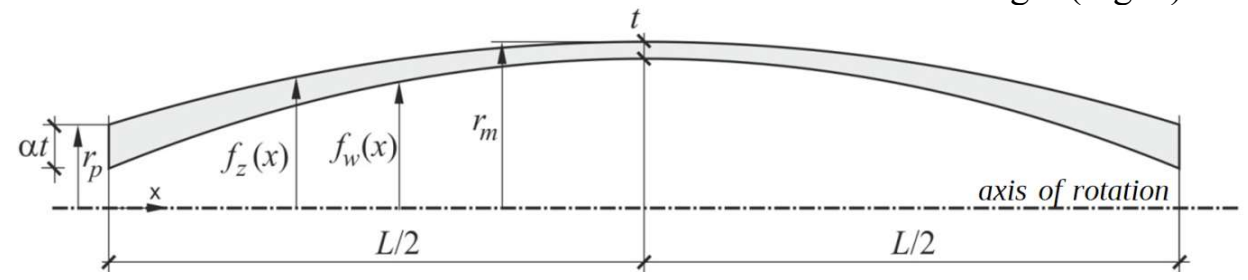

Fig. 4. Cross-section of the upper half of the non-prismatic annular rod and variable wall thickness (scale not preserved; source: [10])

The external and internal generators of rotational surfaces are (as mentioned above) the result of rotation of hyperbolic cosine functions defined as follows:

$$
\begin{gathered}
f_{z}(x)=\frac{\mathrm{r}_{\mathrm{p}}-\mathrm{r}_{\mathrm{m}}}{\cosh \frac{1}{2}-1} \cosh \left(\frac{x}{L}-\frac{1}{2}\right)+\frac{\cosh \frac{1}{2} \cdot \mathrm{r}_{\mathrm{m}}-\mathrm{r}_{\mathrm{p}}}{\cosh \frac{1}{2}-1} \\
f_{w}(x)=\frac{(1-\alpha) \mathrm{t}+\mathrm{r}_{\mathrm{p}}-\mathrm{r}_{\mathrm{m}}}{\cosh \frac{1}{2}-1} \cosh \left(\frac{x}{L}-\frac{1}{2}\right)+\frac{\left(\alpha-\cosh \frac{1}{2}\right) \mathrm{t}+\cos \frac{1}{2} \cdot \mathrm{r}_{\mathrm{m}}-\mathrm{r}_{\mathrm{p}}}{\cosh \frac{1}{2}-1}
\end{gathered}
$$

where $f_{z}(x)$ denotes a functional relation whose graphic image is external forming solids of a bar, while $f_{w}(x)$ - a compound whose graphic image is the inner forming solids of a bar (see Figure 4).

The initial optimization assumption is based on the fact that the non-prismatic CHS bar in question has the same mass with the mass of a certain, solid cylindrical rod of the same length $L$ and cross-sectional radius $\mathrm{r}_{0}$. The additional assumption that both bars will be made of the same amount of material implies their equal volume (Fig. 5); this leads to the relationship:

$$
\pi \mathrm{r}_{0}^{2} \mathrm{~L}=\pi \int_{0}^{\mathrm{L}}\left(f_{z}^{2}(x)-f_{w}^{2}(x)\right) d x
$$


a)

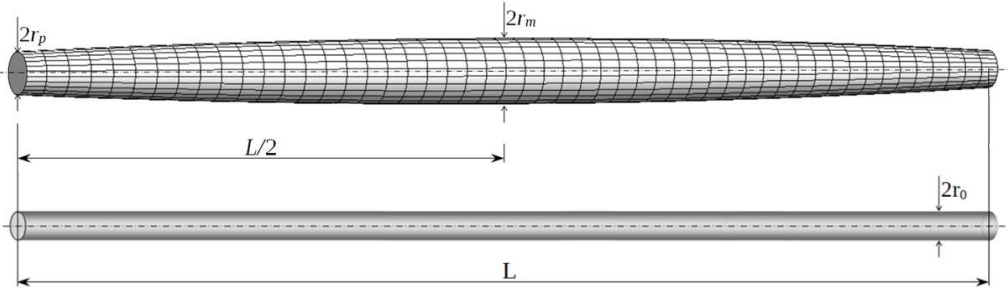

Fig. 5. Rods considered:

non-prismatic CHS bar ( $a$ ) and the reference, solid, cylindrical rod (b)

which, after transformations made in the Mathematica ${ }^{\mathrm{TM}}$ program (cf. [5-6]), binds the radii of individual sections - at the ends of the bar and in its center: $r_{m}=$ $\mathrm{r}_{\mathrm{m}}\left(\mathrm{r}_{\mathrm{p}}\right)$, and namely

$$
\begin{gathered}
\mathrm{r}_{\mathrm{m}}=\frac{(\sqrt{\mathrm{e}}-1)^{4} \mathrm{r}_{0}^{2}+\left(4-2 \alpha+4 e^{3 / 2}(1-2 \alpha) \alpha-\alpha^{2}\right)}{2 \mathrm{t}\left(4-2 e(\alpha-2)+e^{2}(\alpha-2)-\alpha-6 \sqrt{e} \alpha+2 e^{3 / 2} \alpha\right)} \mathrm{t}^{2}+ \\
+\frac{\left(4 \sqrt{e} \alpha(2 \alpha-3)+e^{2}\left(\alpha^{2}+2 \alpha-2\right)+e\left(6 \alpha^{2}-4 \alpha+4\right)\right)}{2\left(4-2 e(\alpha-2)+e^{2}(\alpha-2)-\alpha-6 \sqrt{e} \alpha+2 e^{3 / 2} \alpha\right)} \mathrm{t}+ \\
+\frac{\left(-1+e^{3 / 2}(2-8 \alpha)-\alpha+e^{2}(\alpha+1)+e(6 \alpha-2)+\sqrt{e}(8 \alpha-6)\right)}{4-2 e(\alpha-2)+e^{2}(\alpha-2)-\alpha-6 \sqrt{e} \alpha+2 \mathrm{e}^{3 / 2} \alpha} \mathrm{r}_{\mathrm{p}}
\end{gathered}
$$

where $e$ is the Euler number (also called the Neper number).

Let the considered rod be subjected to axial compression. It was assumed that it is simply supported at the ends and that its geometry was disturbed with the initial, arched geometric imperfection, which is represented by the relation:

$$
f(x)=e_{0} \sin \frac{\pi}{\mathrm{L}} x,
$$

where $e_{0}$ represents the amplitude of imperfection $\left(e_{0}=\mathrm{L} / 250\right.$ adopted in further considerations).

A graphical view of the above assumptions is presented in a static scheme (Fig. 6) in which $w(\mathrm{x})$ denotes an additional bending of the strut.

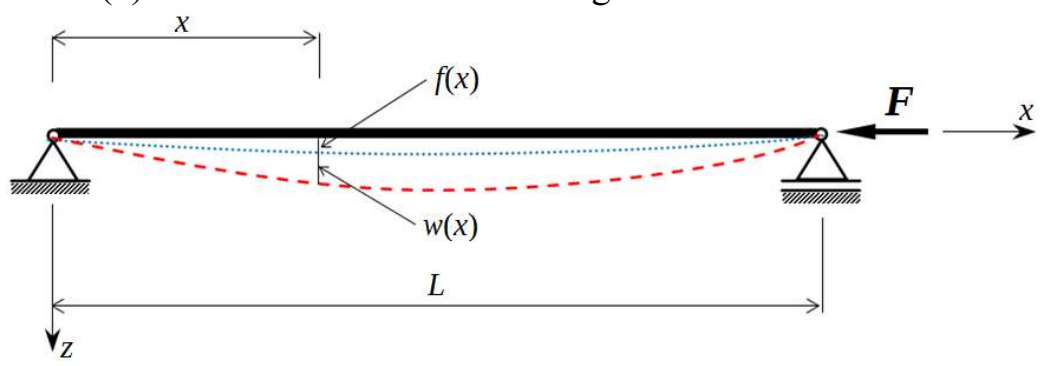

Fig. 6. Static scheme of the system ripening 


\section{OBJECTIVE FUNCTION AND SET OF RESTRICTIONS}

Due to the problem posed, the objective function is the maximum compressive force:

$$
\mathrm{F}=\max
$$

Let us assume that the bar is to work in the elastic range. Therefore, it is necessary to examine the following issue: searching for the maximum compressive force, the value of which will not cause plasticizing the material at any point of the bar $\left(\mathrm{F}_{\text {upl }}\right)$ :

$$
\mathrm{F}_{\mathrm{upl}}=\max \Rightarrow \mathrm{F}_{\text {upl(max) }}=\mathrm{F} .
$$

So, the objective of the optimisation procedure is maximizing the following expression:

$$
F_{u p l}=f_{y} \cdot A\left(\frac{L}{2}\right) \cdot \frac{I\left(\frac{L}{2}\right)}{I\left(\frac{L}{2}\right)+\frac{L}{250} \cdot r_{m} \cdot A\left(\frac{L}{2}\right)}
$$

where:

- $\mathrm{f}_{\mathrm{y}}$ - yield stress of steel used to make the bar,

- $\mathrm{A}(x)$ - variable cross-sectional area:

$$
\mathrm{A}(x)=\pi\left(f_{z}^{2}(x)-f_{w}^{2}(x)\right)
$$

- $\mathrm{I}(x)$ - variable moment of inertia of the cross-section:

$$
\mathrm{I}(x)=\frac{\pi}{4}\left(f_{z}^{4}(x)-f_{w}^{4}(x)\right)
$$

The relationship (3.3) is a consequence of the direct transformation of the relation expressing the stress at the middle section of the rod (Fig. 7) and their limitations by the yield stress $f_{y}$ :

$$
\sigma_{\max }=\frac{F}{A\left(\frac{L}{2}\right)}+\frac{F \cdot e_{0} \cdot r_{m}}{I\left(\frac{L}{2}\right)} \leq f_{y} .
$$

In this formula it was assumed that the amplitude of imperfection bow $e_{0}=\mathrm{L} / 250$ and $\mathrm{F}=\mathrm{F}_{\text {upl }}$ and the inequality was replaced by an equal sign, and after relatively simple transformations the formula (3.3) was obtained.

The same relationship can be obtained by elementary transformation of formula 6.44 found in Eurocode 3 - PNEN 1993.1-1. Steel structure design. General rules and rules for buildings (assuming bending in one direction) (cf. [12]). 
It is worth mentioning that the formula (3.6) does not include the amplification factor $1 /\left(1-\frac{F}{F_{c r}}\right)$ which should increase the initial amplitude $e_{0}$ according to well known Ayrton-Perry approach. This simplification was compensated by the significant increase of the initial amplitude $e_{0}$ to the value $\mathrm{L} / 250$.

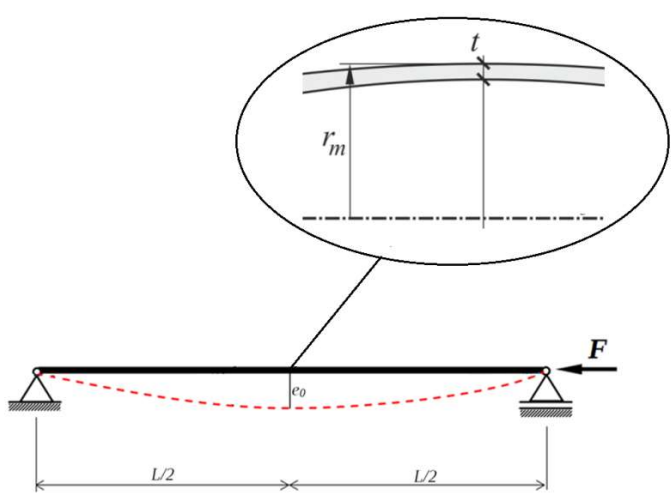

Fig. 7. The geometry of the bar in the middle of its length

Let us move on to bar shape optimization. It will be based on the search for extreme compressive force (3.3) for three independent decision variables:

a) the outer radius forming a cross-section at the ends of the element $\left(r_{p}\right)$,

b) wall thickness at the middle section of the bar length $(t)$

c) the ratio of the wall thickness at the rod ends to the wall thickness at the middle $\operatorname{section}(\alpha)$.

Let us define a set of constraints.

- Geometric constraints:

- equality of bar masses:

$$
m_{p(r)}=m_{p(d)}
$$

where $m_{p(r)}$ - reference bar mass, $m_{p(d)}$ - hollow bar mass,

- positive wall thickness in the middle of the bar length

$$
t>0,
$$

- positive value of the outer radius of the bar solid forming at its end

$$
r_{p}>0,
$$

- positive value of the wall thickness ratio at the rod ends to the wall thickness in its half

$$
\alpha>0,
$$


- Stress limits:

- the maximum stress should not exceed the yield stress $\left(\mathrm{f}_{\mathrm{y}}\right)$ at the end of the bar and half its length:

$$
\sigma_{\mathrm{rz}}(0) \leq \mathrm{f}_{\mathrm{y}}, \quad \sigma_{\mathrm{rz}}(\mathrm{L} / 2) \leq \mathrm{f}_{\mathrm{y}},
$$

- the impossibility to exceed the critical stress values of the cylindrical shell (local loss of stability of the cylindrical compression shell; cf. [7, 17-20]) at the end of the bar and half its length:

$$
\sigma_{\mathrm{rz}}(0) \leq \sigma_{\mathrm{gr}(\mathrm{p})}(0), \quad \sigma_{\mathrm{rz}}(\mathrm{L} / 2) \leq \sigma_{\mathrm{gr}(\mathrm{s})}(\mathrm{L} / 2),
$$

where symbol $\sigma_{\mathrm{rz}}$ means the actual stresses in the bar (more precisely in the bar wall), while the limit stresses are described by relations

$$
\begin{gathered}
\sigma_{\operatorname{gr}(\mathrm{p})}(0)=\frac{1}{\sqrt{3\left(1-v^{2}\right)}} \frac{\mathrm{E \alpha t}}{R(0)^{\prime}} \\
\sigma_{\operatorname{gr}(\mathrm{s})}(\mathrm{L} / 2)=\frac{1}{\sqrt{3\left(1-v^{2}\right)}} \frac{\mathrm{Et}}{R(\mathrm{~L} / 2)}
\end{gathered}
$$

where:

$$
R(x)=\frac{1}{2}\left(f_{z}(x)+f_{w}(x)\right),
$$

and $E$ is Young's modulus.

It is worth of mentioning that the formula (3.13) is valid for the compressed cylinder of constant wall thickness. It was adopted here to eliminate the wall local buckling.

The search for extreme (more precisely maximum) compressive force (objective function defined by formula (3.3)) was implemented in the Mathematica ${ }^{\mathrm{TM}}$ program (cf. [6, 7]). This operation is fully implemented in the Maximize procedure (allowing to find the maximum global function in the area defined by constraints), although the problem raised, due to the complexity of calculations, uses the equivalent command using the iterative (numeric) sequence-NMaximize , which performs the same task. It was requested that the iterative array uses a stochastic optimization method, namely the differential evolution algorithm (Differential Evolution, cf. [6,13]). The number of iterations was limited to 500 . As it results from the above, determining the shape of a bar with maximum load carrying capacity involves carrying out a highly complicated optimization procedure that can be carried out in a program using symbolic transformations for example, in the referenced Mathematica ${ }^{\mathrm{TM}}$ program. Due to the fact that it is a very advanced program and hardly accessible to engineers or designers, there has been a need to adapt the results of complex optimization procedures to project 
needs, by creating a relatively simple algorithm that can be used in engineering practice.

The performed analysis (a number of calculations and numerous observations supported by many examples) showed that it is possible. Below is the algorithm that allows to determine the shape of an optimal bar, i.e. a bar showing increased resistance to compression.

More precisely, the bar geometry presented by the formulas (2.1) and (2.3) is complicated due to the complexity of these relations, especially (2.3), which is a consequence of the equation (2.2). This causes a lot of trouble in engineering calculations. The procedure shown below is a presentation of the algorithm that will best approximate the exact procedure, while being simple and easy to apply. The simplification consists in replacing the relation (2.3) with its approximate form - the formula (3.19) and using empirical relations (3.20) and (3.21), which allow to determine the parameters sought. Thanks to all measures, the increase in load capacity practically does not decrease and the calculations become much easier.

The proposed design procedure obtained as a result of very laborious optimizations carried out by means of Mathematica ${ }^{\mathrm{TM}}$ system is as follows.

The procedure is confined to reference bars of following geometrical parameters:

- radius of the cylindrical reference bar [mm]:

$$
4 \leq \mathrm{r}_{0} \leq 40,
$$

- slenderness of the cylindrical reference bar:

$$
\lambda=2 \mathrm{~L} / \mathrm{r}_{0} \text { and } 100 \leq \lambda \leq 250,
$$

which implies a condition that meets the length of the bar:

$$
\frac{100 \mathrm{r}_{0}}{2} \leq \mathrm{L} \leq \frac{250 \mathrm{r}_{0}}{2}
$$

The outer radius forming a cross-section of optimal bar at its ends (value adopted a priori):

$$
\mathrm{r}_{\mathrm{p}}=\mathrm{L} / 25 \text {, }
$$

- outer radius forming cross-section at the middle of the length of the bar:

$$
r_{m}=1,45 r_{p}
$$

- the ratio of the wall thickness of the support member at its ends to the wall thickness of the element at the middle of its length: 


$$
\begin{gathered}
\alpha(\lambda)=1738143 \cdot 10^{-6}-11228 \cdot 10^{-6} \lambda+11567 \cdot 10^{-8} \lambda^{2}+ \\
-61091 \cdot 10^{-11} \lambda^{3}+16194 \cdot 10^{-13} \lambda^{4}-17067 \cdot 10^{-16} \lambda^{5},
\end{gathered}
$$

- wall thickness in the middle of the length of the supporting bar element:

$$
\begin{aligned}
t(\lambda) & =\left[41167 \cdot 10^{-5}-69305 \cdot 10^{-7} \lambda+478 \cdot 10^{-7} \lambda^{2}+\right. \\
& \left.-1520808 \cdot 10^{-13} \lambda^{3}+1842424 \cdot 10^{-16} \lambda^{4}\right] \mathrm{r}_{\mathrm{m}}
\end{aligned}
$$

Compressive force value $\left(\mathrm{F}_{\mathrm{wrz}(\max )}\right)$, sustained by the bar defines the relation (3.3). The increase in the load carrying capacity of the optimal spindle rod (defined as above) in relation to the load capacity of the reference rod [\%] is determined by the formula:

$$
\mathrm{W}_{\text {wrz-ref }}=\frac{\mathrm{F}_{\mathrm{wrz}(\max )}-\mathrm{F}_{\text {ref(max })}}{\mathrm{F}_{\text {ref(max })}} \cdot 100
$$

where: $F_{\text {ref(max) }}$ is the maximum compressive force of the reference bar:

$$
F_{\text {ref(max })}=f_{y} A_{0} \frac{I_{0}}{I_{0}+\frac{L}{250} r_{0} A_{0}}
$$

and $\mathrm{A}_{0}$ and $\mathrm{I}_{0}$ is the cross-sectional area and the moment of inertia of the reference bar, respectively.

The relation (3.19) and relationships (3.20) and (3.21), which are functions of the slenderness of a reference bar and have the character of polynomial expansions, are the result of the analysis and interpretation of the results obtained for many cases of bars of various shapes - a number of reference bars for which geometries were defined were adopted the following slenderness $(\lambda)$ and radii $\left(\mathrm{r}_{0}\right)$ :

$-\lambda=\{100,125,150,175,200,225,250\}$,

- $\mathrm{r}_{0}=\{4,5,8,10,20,30,40\}[\mathrm{mm}]$

and the materials from which they were made:

- steel S $235\left(\mathrm{f}_{\mathrm{y}}=235 \mathrm{MPa}\right)$,

- steel S $275\left(\mathrm{f}_{\mathrm{y}}=275 \mathrm{MPa}\right)$.

Studies have shown that in each case analyzed:

- ratio $r_{m} / r_{p}$ is constant and almost equal to 1.45 - hence its approximate value (cf. (3.19)),

- dependencies (3.20) and (3.21) are the result of statistical polynomial regression: fifth polynomial in the case of $\alpha=\alpha(\lambda)$ (formula (3.20)) and fourth-degree polynomial for formula $t / r_{m}=t(\lambda) / r_{m}$ (relation (3.21)); the choice of the degree of individual regression polynomials was dictated by the 
value of the correlation coefficient (R), which, in each of the analyzed cases, was in the range $0,99 \leq \mathrm{R} \leq 1$.

On the basis of the results obtained and the statistical regression carried out, it can be stated that the use of the formula (3.19) and the obtained functional relations (3.20) and (3.21), and thus the entire algorithm (3.15) - (3.23), is completely justified. To be sure that the simplified form of the formula (3.3) is justified, it is recommended to check if $\mathrm{F}_{\mathrm{ult}} / \mathrm{F}_{\mathrm{cr}}<0.7$, where $\mathrm{F}_{\mathrm{cr}}$ is the buckling load determined from the energy criterion for the considered bar (cf. [9-11]). If the condition $\mathrm{F}_{\mathrm{ult}} / \mathrm{F}_{\mathrm{cr}}<0.7$ is fulfilled, the amplification factor is equal 1 and the formula (3.3) is fully correct. In opposite case it is recommended to recalculate $F_{\text {ult }}$ from formula (3.3) in which the amplification factor is taken into account.

\section{NUMERICAL EXAMPLE}

As an example let us try to determine the optimal shape of the cylindrical reference bar with a radius of $18 \mathrm{~mm}$ and a length of $1184 \mathrm{~mm}$, determine the shape of the optimal bar, sustaining the maximum compressive force. The material from which the bar was made - steel S235 ( $f_{y}=235 \mathrm{MPa}$ - according to PN-EN1993-1-1).

Data:

Reference bar:

- radius $\mathrm{r}_{0}=18 \mathrm{~mm}$,

- length $\mathrm{L}=1184 \mathrm{~mm}$.

Geometric characteristics of the reference bar:

- slenderness $\lambda=131,55$,

- cross-sectional area: $\mathrm{A}_{0}=1017,9 \mathrm{~mm}^{2}$,

- moment of inertia of the cross section: $\mathrm{I}_{0}=82448,0 \mathrm{~mm}^{4}$,

- value of maximum compressive force: $\mathrm{F}_{\text {ref(max) }}=116,544 \mathrm{kN}$.

The solution is presented in Table $1 \mathrm{in}$ which results of the proposed simplified procedure were compared with the strict optimization results.

Table 2. Comparison of optimal rods, obtained by using strict and proposed algorithms

\begin{tabular}{|c|c|c|}
\hline & $\begin{array}{c}\text { Optimal rod according } \\
\text { to strict optimization } \\
\text { procedure }\end{array}$ & $\begin{array}{c}\text { Optimal rod according } \\
\text { to the proposed } \\
\text { algorithm }\end{array}$ \\
\hline$r_{\mathrm{p}}$ & $49.90 \mathrm{~mm}$ & $47.36 \mathrm{~mm}$ \\
\hline$r_{\mathrm{m}}$ & $71.60 \mathrm{~mm}$ & $68.67 \mathrm{~mm}$ \\
\hline$\alpha$ & 1.28 & 1.29 \\
\hline $\mathrm{t}$ & $2.37 \mathrm{~mm}$ & $2.48 \mathrm{~mm}$ \\
\hline$F_{\text {ult }}=F_{\text {wrz (max) }}$ & $216.551 \mathrm{kN}$ & $216.059 \mathrm{kN}$ \\
\hline $\mathrm{W}_{\text {wrz-ref }}$ & $85.81 \%$ & $85.39 \%$ \\
\hline
\end{tabular}


The solution is presented in Table 1 in which results of the proposed simplified procedure were compared with the strict optimization results.

To be sure that the amplification factor could be neglected, the critical force was calculated. From the energetic criterion the value $F_{c r}=18.0325 * 10^{6} \mathrm{~N}$ was obtained. It means that the amplification factor is equal

$$
\frac{1}{1-\frac{F}{F_{c r}}}=\frac{1}{1-\frac{0.216059 \cdot 10^{6}}{18.0325 \cdot 10^{6}}}=1,012 .
$$

It confirms the correctness of the simplified form of the formula (3.3). The shape of the rod showing maximum resistance to compressive force is shown in Fig. 8. In authors' opinion, the proposed simplified algorithm for determining the optimal shape of a compressed bar is almost consistent with the exact algorithm. This fact is confirmed by the above example. The differences between the results of the strict optimization procedure and the results of the proposed, simplified approach are small and confirm the usefulness of the latter for practical engineering calculations.

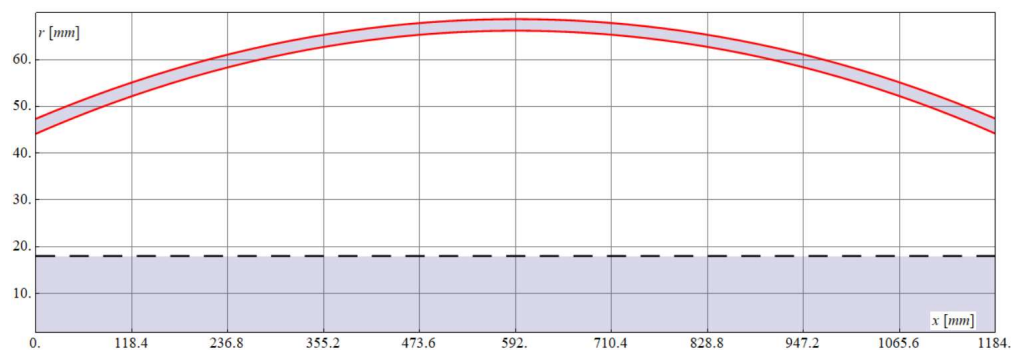

Fig. 8. Graphic image of the upper half wall of the optimal rod and the upper part of the reference rod - under the dashed line

(scale not preserved)

\section{SUMMARY AND CONCLUSIONS}

The procedure for designing of steel rods exhibiting maximum compression resistance proposed in the work is effective and possible to use in engineering practice.

1. The advantage of the proposed shape of the bar is that it allows to increase the value of its load carrying capacity, i.e. it ensures the transfer of a higher value of compressive force than similar structural member of the same mass and length. The extent of the increase in the load capacity relative to the load capacity of the reference, solid cylindrical bar depends on the slenderness of the reference bar and ranges from $60 \%$ to $170 \%$. 
2. Due to this very beneficial fact, the proposed design procedure can be used wherever it is required to maintain a certain stiffness and an increased value of compressive force is desired, as well as in constructions where it is necessary to reduce weight while maintaining the adopted mechanical parameters, e.g. values compressive force.

3. The compressive bar was shaped in such a way that under applied compressive force, the magnitude of normal stresses did not exceed the values at which elastic-plastic deformations could occur. The proposed shape also guarantees the rod's resistance to local buckling.

4. One of the many uses of the support bar element according to the authors' proposal may be to place it in the position of the strut of the shock absorber cups in the engine compartment of the car. It is also possible to use a support rod as a core of a beam with a smaller mass compared to known spreaders. It is possible to make compressed truss elements in the shape proposed. In this way one can also shape simply supported columns in building structures. All the cited applications allow to significantly reduce the mass of the assembly in which the compressed bar elements occur. ripening.

\section{REFERENCES}

1. Brandt, AM et al. 1984. Criteria and Methods of Structural Optimization. PWN - Polish Scientific Publishers, Warszawa.

2. Bródka, J and Łubiński, M 1971. Light steel constructions. Arkady Publishing House, Warszawa.

3. Brzoska, Z 1965. Statics and stability of rod and thin-walled structures. State Publishing House, Warszawa.

4. Eulero, L 1774. Methodus inveniendi lineas curvas maximi minimive proprietate gaudentae, sive solution problematic isoperitrici lattesimo sensu accepti. // Additamentum 1: De curvis elasticis. Lusanne and Geneva, Apid Marcum-Michaelem, Bousquet et Socios.

5. Glabisz, W 2003. Mathematica in structural mechanics. Oficyna Wydawnicza Politechniki Wrocławskiej, Wrocław.

6. Gliński, H et al. 2012. Mathematica 8. Publishing House of the Jacek Skalmierski Computer Laboratory, Gliwice.

7. Huber, TM 1958. Technical stereomechanics. State Publishing House, Warszawa.

8. Lagrange, JL 1770 - 1773. Sur la figure des colonnes. Miscellanea Taurinensia.

9. Marcinowski, J and Sadowski, M 2016. Buckling capacity of nonprismatic rods with polygonal cross-sections. In: Sustainable 
construction, the University Publishing House of the University of Technology and Life Sciences in Bydgoszcz, Bydgoszcz.

10. Marcinowski, J and Sadowski, M 2015. Shape optimization of nonprismatic rods of circular hollow cross-sections and of variable wall thickness. W: Proceedings of the stability of structures: XV-th symposium. Zakopane, Polska, 2018. Łódź: Katedra Wytrzymałości Materiałów i Konstrukcji Politechniki Łódzkiej, 99-100.

11. Marcinowski, J and Sadowski, M 2020. Using the ERFI Function in the Problem of the Shape Optimization of the Compressed Rod. International Journal of Applied Mechanics and Engineering, Vol. 25, no. 2, 75-87.

12. PN-EN 1993-1-1 2006. Eurocode 3: Design of steel structures. Part 1-1: General rules and rules for buildings. PKN, Warszawa.

13. Opara, K 2014. Analysis of the differential evolution algorithm and its application in the determination of statistical dependencies. Abstract of the phD thesis.

14. Promotional article Innovative joining techniques in lightweight EJOT ${ }^{\circledR}$ constructions set trends, (http://ejot.pl/p1/download/Ejot_art_STA5_10.pdf).

15. Romanów, F 1992. Stability of structures. University of Engineering Publishing House, Zielona Góra.

16. Rykaluk, K 2012. Problems of metal structure stability. Dolnośląskie Wydawnictwo Edukacyjne, Wrocław.

17. Timoshenko, SP and Gere, JM 1963. Theory of elastic stability. Arkady Publishing House, Warszawa.

18. Timoshenko, SP and Goodier, JN 1962. Theory of elasticity. Arkady Publishing House, Warszawa.

19. Timoshenko, SP and Voinowsky-Krieger, S 1962. Theory of plates and coatings. Arkady Publishing House, Warszawa.

20. Thompson, P, Papadopoulou, G and Vassiliou, E 2007. The origins of entasis: illusion, aesthetics or engineering? Spatial Vision, Vol. 20, No. 6, 531-543.

21. Wołgin, LN 1970. Optimization. Scientific and Technical Publishing House, Warszawa. 